\title{
Cost Estimation in Initial Stages of Product Development - An Ontological Approach
}

\author{
Rafael VOLTOLINI ${ }^{\mathrm{a}, 1}$, Milton BORSATO ${ }^{\mathrm{a}}$ and Margherita PERUZZINI ${ }^{\mathrm{b}}$ \\ ${ }^{\text {a }}$ Federal University of Technology - Paraná (UTFPR) \\ Rua Dep. Heitor Alencar Furtado, 5000 - 81280-340 - Curitiba, PR, Brazil \\ ${ }^{\mathrm{b}}$ University of Modena and Reggio Emilia (UNIMORE) \\ Via Vivarelli 10, 41125 - Modena, Italy
}

\begin{abstract}
Cost estimation in the early stages of the product development process is fraught with uncertainties. The conceptual design is characterized by the absence of data, the most critical being costs. Decisions based on incorrect assumptions impact a project significantly and can increase unexpected costs in the future. As there are no structured means of obtaining costs in the conceptual phase, the reuse of data from past projects is an alternative discussed in the literature. Knowledge management approaches suggest a search for data in successful earlier projects. The use of ontologies has been regarded as an approach to capturing either knowledge stored in database or tacit knowledge. The proposed solution, in the form of an expert system built upon an ontological model, seeks to estimate costs based on costs in previous projects as well as expert tacit knowledge. The model is demonstrated by queries with needed functions and requirements. The ontological model searches the necessary information and generates a cost estimation. The present research project follows the methodological framework Design Science Research, presenting an overhead crane as a case study. The proposed approach has great potential in other industrial contexts as well.
\end{abstract}

Keywords. Expert systems, Ontology, Cost estimation, Product development.

\section{Introduction}

New Product Development (NPD) professionals constantly deal with uncertainties in the initial stages [1]. With the accomplishment of each gate, the product data is organized, its features evolve and become clearer towards the intended product. Different authors warn about one of the most dangerous uncertainties that a project can handle: costs [2]. The challenge in the initial stages is to estimate product cost under a scenario with unclear customer requirements. There is an information gap in the conceptual phase where the analysis is complex [3].

The cost estimation process is composed of implicit estimations mostly, such as customer's satisfaction, configurations of the project, or similarities of the requirements [4]. Professionals estimate the costs considering the concepts acquired in past experiences. Experts with years of experience have more knowledge and chance to assert the final cost. Despite the difficulty to dispose of experts in cost estimation, an artifact to reproduce the great capabilities may support in initial stages. The closer to the initial phases the less assertive is the cost estimation. In the detailed project, the cost specialist

\footnotetext{
${ }^{1}$ Corresponding Author, Email: voltolini@alunos.utfpr.edu.br.
} 
has clearly requirements and tools to develop a structured estimation. Exist a knowledge gap in the initial stages where decisions are based on assertions. An artifact based on an expert system could simulate the professionals knowledge [5]. Estimating costs right the beginning of the process when the requirements are unclear. Any question could be answered based on past projects and knowledge reuse.

Previous researches in the ontology domain are broad and demonstrate dynamism in the applications. [6] present a literature review of ontologies focused on inference where exposes the knowledge capture, instantiations or mapping are still manually and aims to reduce product costs. In the construction field, there is an ontology for cost estimation in construction coatings to capture the tacit knowledge and return a unique solution [7]. The tool developed by [8] is able to facilitate knowledge management and to reuse structure for product-service systems to optimize projects and reduce costs. Every data influence on cost estimation in an unique way. In order to manage this cluster of information, [9] and [10] present alternatives to cost estimation, but they are dependent on customer's requirements to generate a graphical model. The tool LeanCOST developt a cost estimation based on the CAD model and consider data acquired in the detailed stages. In these approaches, the cost estimations are dependent on information not always available and not instantaneous. [11] present a Knowledge-Based Systems (KBS) approach to increase the level of knowledge reuse in ontology engineering disciplines. [12] presented a cost estimation method to reduce the costs in the manufacturing system of an automated vehicle. [13] discuss a cost estimation model using artificial intelligence techniques for new products through the development stages. Other applications aim to estimate the costs in construction field supporting the specialists [14]. The main researches avoid the initial stages under unclear data, which opens possibilities in the state of art.

This research aims to present an ontological model to support the cost estimation in the initial phases. Based on previous projects and human expertise the model estimates the costs of similar products. The reuse of knowledge allows the specialists to handle lots of information and resolve complex analysis of data.

This paper is structured in four sections. First, the introduction presents the context; in the second, the methodological aspects are described; in the third, the ontology development is presented with examples to understand the artifact functionality; the fourth section contains the discussions and finally the research conclusion.

\section{Methodological aspects}

Based on Design Science Research (DSR), a methodological framework, the research is prescriptive and aims to deliver a solution to the problem identificaion. It is divided into six stages: i) problem identification and motivation, ii) objectives definition, iii) artifact creation, iv) demonstration, v) evaluation and vi) communication. The method Ontology Development 101 was followed to develop the artifact [15].

According to Method 101, the first step is the problem identification, which was presented in [16]. The research pointed out the difficulties to deal with uncertainties at the beginning of the project. The initial stages are characterized by ambiguity to deal with parameters since there are few data about the customer requirements. When the engineer develops a cost estimation, without data, the result is undefined and full of uncertainties. An ontological approach was suggested to capture and reuse knowledge from specialists in past projects. After the objectives definition, the development of the 
artifact followed method 101. Although different alternatives for developing ontologies such as Ontoanimal [17], TOVE [18], OntoTracED [19] and Methontology [20], method 101 is recommended by Protégé team from Stanford as a guide to create the first ontology. The demonstration carried out through the case study of the product overhead crane. As indicated by the method, competency questions must be created in the beginning to be answered by the ontology in the end. The evaluation follows through the method FOCA and the demonstration is represented by this paper.

\section{The ontological model}

The model development origin in the functional concept. The project requirements are translated to a functional representation. It is an alternative to use tacit knowledge, compound by implicit knowledge, and translate into explicit rules. The product functions are a formal knowledge as product documents, computer algorithms, and intelligent knowledge systems [21]. The ontology accepts all possible costs found in the initial stages of the product development. The model can be improved to specific costs if the developer guide this path.

The case study describes an overhead crane. It is a consolidated product in the industry with fewer modifications in the last years. Innovations are incremental and represent project improvement according to the application and customer requirements. Few parts are represented by the ontology, most of them the main components of the module.

Figure 1 presents the class hierarchy, with the top classes Artifact, Cost, Function, and Specification. Artifact means the product described by the case study. During the development process, the subclasses changes as the developer abstractions changes as well. The idea is to separate the final product in a few groups to represent all equipment. All classes belonging to Artifact are physical components of a product, produced or bought. Cost is responsible to describe the cost estimation of a product. ProductCost is the class representing the costs with product metric, identified by the quantity of what is consumed by each marketed item. Productioncost brings the concepts of costs related to the production volume, represented by FixedCost and VariableCost. Functions represents the main functions of the equipment overhead crane. Specification means other characteristics which compose the artifact, they are Date, Distance, Evaluation, Load and Speed.

The product is divided into modules according to the axis it moves: Bridge, Trolley, Hoist. The module Bridge means the structure formed by the beams that sustain the overhead crane. It moves in the y-axis running through the support columns of the shed. Trolley means the cart responsible for pulling the loads, which includes the motor, the reducer, the drum, etc. It moves in the $\mathrm{x}$-axis running across the bridge to moves the load from one point to another. Then, Hoist is the module responsible to lift the load, compound by the rope, the hook, etc. It moves in the z-axis lifting the load until the beam height.

In the ontology, the product has two main classes to represent the prior functions: ToLift and ToMove. ToLift is connected to the movement in the z-axis, while ToMove can be represented by $\mathrm{x}$ and $\mathrm{y}$-axis. The axes have a connection with the assembly's movement by functions. The assembly Trolley moves only in the x-axis 
and connects to other classes by the function ToMove. Bridge moves in the y-axis and shares the function ToMove with the Trolley. Hoist is the only assembly with relation to the function ToLift moving toward the z-axis.
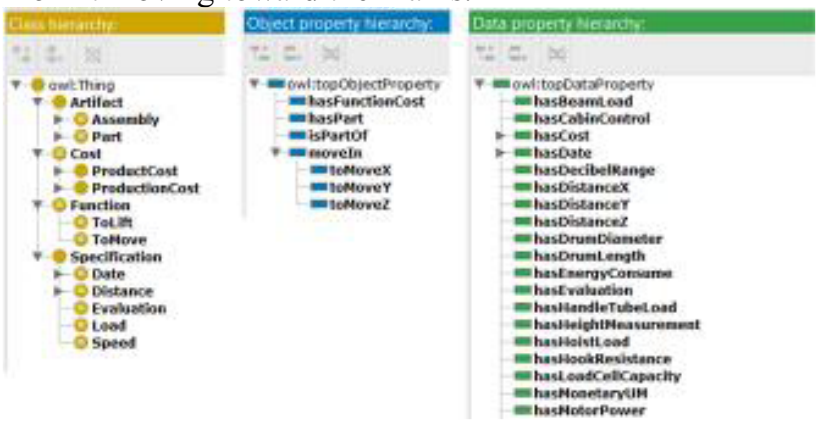

Figure 1. Ontology structure in Protégé

The demonstration is performed by using queries with Sparql in Protégé. The first query is presented in Figure 2. It can be presented in artificial or natural language. In natural language, the query asks for the cost of an item, the motor. The query is not specifying any model variation, then the answer has to return all costs of all items. The artificial language follows a different approach. The first stage is to declare the prefix, the scheme used in the query [22]. It appoints the variable ?t and says it has a part which belongs to the variable ?motor. ?motor is still an empty slot. Then, the query states ?motor has a power motor with the data property FC:hasMotorPower. The variable ?power is just auxiliary. Also, the query says ?motor has a certain cost with the data property FC:hasCost. The cost is called by the variable ?cost. The results are the individuals called Motor 01 and 02 and the costs. The final cost is the number one due to the strategy to use symbolic value to simulate the application.

The cost estimation could contain actual industry data. But this process takes time and availability from both the industry and the researcher. The decision to use illustrative data cooperated with the restricted timeline. Each instance that represents a cost to the assembly received the value of a single monetary unit. A product with 5 components costs 5 monetary units, as well as a product with 1000 items costs 1000 monetary units. To simulate the cost estimation this approach collaborated with the Protégé fast compilation and satisfactory results for the artifact understanding. In real industrial cases, it is necessary to change the values of the instances slots, which does not reduce the applicability of the model.

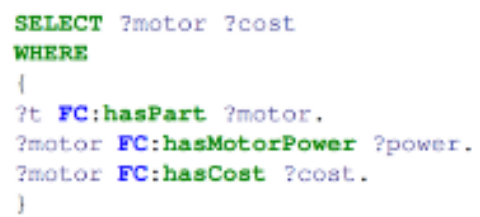

Figure 2. Query \#01 - How much is the motor?

The next query has the intent to request an assembly which the motor is part of. Figure 3 exposes ?t has a part - a component - containing property of motor power. Still, it is part of an assembly. The result is sorted in ascending alphabetical order. Figure 4 presents the ontology has two motor variations belonging to three different assemblies. It indicates a motor can be part of different assemblies. TrolleyStructure is an instance representing an assembly if it has a different component inside it is characterized as a different assembly. 


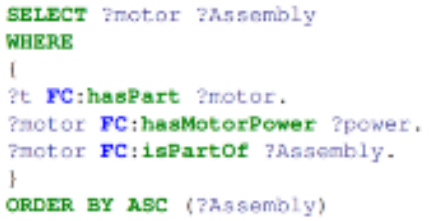

Figure 3. Query \#03 - Which assembly has a motor item?

\begin{tabular}{|c|c|}
\hline Pmotoe & Tassenth \\
\hline FCMotoro1 & FCTrolleystructure01 \\
\hline FCMotoro2 & FCTrolleystructure 02 \\
\hline FCMotoro2 & FCTrolleystructure 02 \\
\hline FCMotoro2 & FCTrolleystructure 03 \\
\hline FC:Motor02 & FC.Trolleystructure 03 \\
\hline
\end{tabular}

Figure 4. Result of the Query \#03

Figure 5 asks for the sum of part costs of the assembly TrolleyStructure03. The function SUM delivers the final result in the variable ?total. Figure 6 present the result seven because this is the number of parts belonging to the assembly (Drum03, SpecialPainting, Motor02, Reducer02, SevereUse, AnticollisionDetector, PulleyCompensationHoist02).

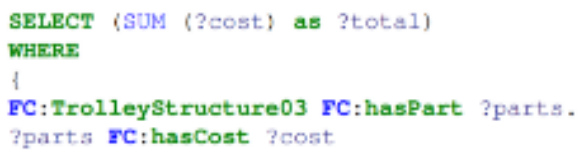

Figure 5. Query \#05 - What is the cost of the assembly TrolleyStructure03?

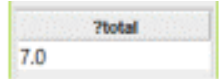

Figure 6. Result of the Query \#05

An ontology has different alternatives to be represented, it depends on the plugin or software used. Assuming a different approach to understand the ontology content, WebVOWL [23] presents the graph with connections to achieve the result. The objective of this approach is to show the logic behind the ontology. The equipment overhead crane has mostly two main functions. To lift a load from the inertia and to move the load through a distance by the field plane. Figure 7 focus on the relation between the equipment and the functions. Analyzing from the bottom, Function has two subclasses, ToLift, and toMove. The only alternative to performing a given function is through the axis $x, y$ or $z$. Following the structure logic, all assemblies are connected to a specific axis. In the end, they belong to the Assembly which has a Part. Thus, the equipment has a close connection to the functions.

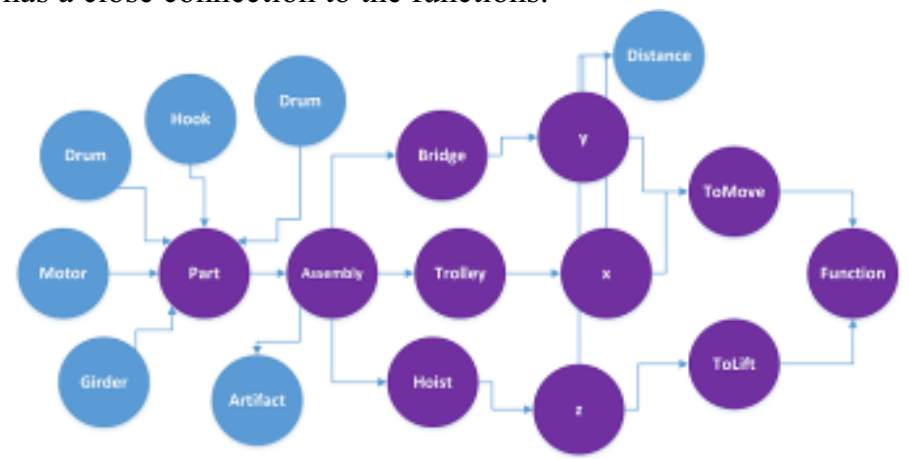

Figure 7. Query \#08 - Which is the connection between functions to the equipment? 
Now, joining the two approaches the result is enlightening. Figure 8 brings the question referring to a specification. It uses the data property hasHoistLoad because the instance HoistStructure has an evaluation integrated. The hasEvaluation is used to draw the evaluation. This query is accompanied by both alternatives answers. Figure 9 presents the results with grades varying between 4 and 5. The benefits of using grades when the knowledge is reused rely on avoiding mistakes from past projects. Results without any register do not appear. Using WebVOWL, Figure 10 presents the connections. The Evaluation is a sub-class of Specification connected to the axis $z$ by the class Distance. As requested by the query, the z-axis is connected to the class Hoist, which is an assembly compound by parts defining an artifact. The function linked to the $\mathrm{z}$-axis is ToLift, always related to costs.

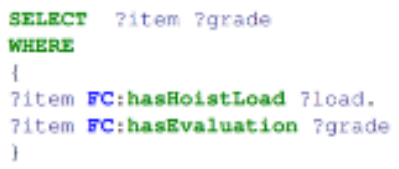

Figure 8. Query \#09 (algorithm structure) - Is there a performance feedback from an assembly in past sales?

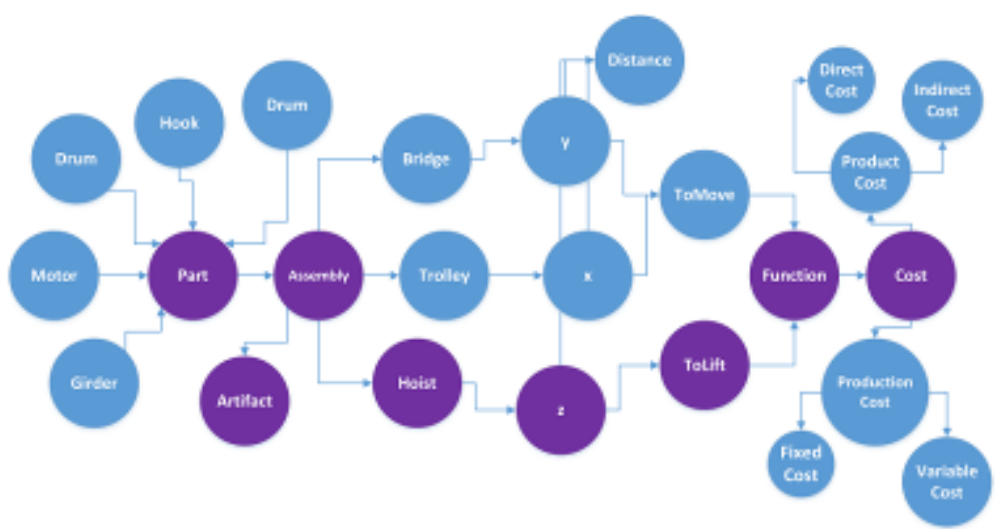

Figure 10. Query \#09 (visual structure) - Is there a performance feedback from an assembly in past sales?

The ontology knowledge-based reuse data from past projects. However, if the query refers to a new case not described in the database, the ontologist could have difficulties to find the answer. A new case represents different applications or the customer requiring two overhead cranes synchronized. Is it possible to develop for the first time? The answer is yes, despite leak of explicit knowledge in the ontology database. The company can consider an instance with a cost to develop new projects. Searching for data is also an ontology advantage. It is not a faster solution but acts as a support.

The competency questions represent a customer asking for cost estimation of the product overhead crane. The classes, instances, and properties are created exclusively to answer the competency question. Abstractions beyond what is required may confuse the developer. The abstraction depth level must be capable of answering the competency 
question. Follow the competency questions: How much does it cost to hoist the load X1, with two lifting speeds X2 and X3, moving the load with speeds X4 and X5, in lateral and longitudinal directions, respectively? Also, it is required a robust body, remotecontrolled device to handle tubes and collision sensors. The shed has the following measures: $\mathrm{W} \times \mathrm{L} \times \mathrm{H}$ meters. The strategy used in the ontology was to change all costs to one (01) monetary unit. The result delivers the cost and also the number of parts compounding the product assembly. The ontology was able to answer the query, achieving the goal established. Then, the evaluation must verify how well the artifact satisfied the objective.

\section{Ontology evaluation}

The evaluation means to test the artifact on solving a problem. While in the demonstration the artifact needs to show that it meets the objectives, in the evaluation phase it is necessary to prove that it serves well. The ontology must answer the questions satisfactorily. [24] suggest relevant metrics and analysis techniques. The researcher has to decide if the artifact satisfies the objectives or if it is necessary to return to the initial stages to solve problems. Improve the development according to the metrics chosen is an iterative process. The artifact evaluation was conducted with the FOCA, which is a method to evaluate ontologies based on quality criteria.

While DSR collaborates in all work development, the FOCA seeks to assist in the development of the ontological artifact. From the objectives to be achieved, the ontology receives grades according to certain metrics, such as completeness, adaptability, consistency, among others. Although different ontology evaluation alternatives have criteria, they depend on the evaluator's experience and may, therefore, be imprecise.

Considering external opinions to evaluate the ontology, FOCA suggests specialists on ontology and/or on the domain, in this case, costs. The scenario was presented by a video and the questionnaire available by Google Forms reproducing the questions defined by the ontology evaluation authors. Attached, four competency questions in natural and artificial language were sent to exemplify the ontology functionality. The questionnaire was sent to 08 ontology specialists, with 06 replies. None of the interviewed had specific knowledge about cost domain, just superficial. It was not sent to cost specialists due to the difficulty to comprehend the computational language. A future evaluation must consider it. Figure 11 presents the result of the quality verification based on the FOCA general result. Specialist \#05 indicated the lowest grade compared to the other specialists. Following the FOCA statistical equation, the results require an approximation to expose differences between interviewers. The grades can vary from 0.25 to 0.99 .

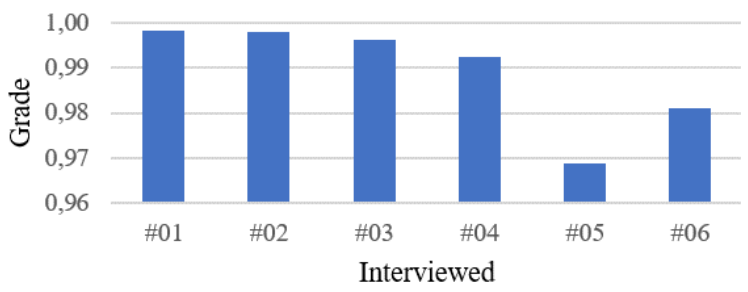

Figure 11. FOCA results - Quality verification 
All recommendations are based on OWL file version 17, that originated the version 18. It was suggested new queries to construct the knowledge to the interviewed understand and visualize better. Starting from simple queries and elevating the complexity until the competency questions. Missing annotations were corrected to avoid misunderstanding by the evaluators. The introduction received improvements to represent better the domain and case. In general, the domain was clear to most part of participants and the ontology was objective to what was specified.

Following the FOCA steps, after the general quality evaluation, partial quality criteria evaluates specifics roles. The first is the substitute, which means how the ontology describes the real world by concepts, as closer the concept to the real world better. The interviewed \#05 pointed the competency questions were not enough. It was suggested to create queries to be applied before and construct the knowledge until the final competency question as presented in this research. The reuse of other ontologies was unclear to some interviewed. The suggestion was to use the Active Ontology tab to inform the user about the content as clear as possible. In this case, the ontology version 18 has an introduction in the first tab satisfying the suggestion.

The role ontological commitments create a relation to the real world, how similar the ontology is. A good grade means consistency in the representation, a high level of abstraction. It was suggested to interview more domain specialists to fortify the cost domain. More insertions about the product could also increase the commitments.

The intelligent reasoning means how the ontology infers the world, how the relations and attributes are defined. Specialist \#01 commented on the use of terms shared with different domains. For example Drum, it can be used as a part of an overhead crane and a musical instrument. The annotations were revised to clarify the user about the content. More consistency could be achieved by increasing the content about the product and cost domain.

The efficient computation represents how the ontology can think about a domain in computer applications. We tested different reasoners (Pellet, HermiT, Ontop). Pellet was faster been used during the development process. To answer the questionnaire, it was suggested this reasoner to achieve satisfactory grades. As only one interviewed evaluated with a low grade, this case was disregard because could represent a local case or incapable machine. The human expression, the fifth goal, means how easy it was to understand the modeling. All grades were $75 \%$ or $100 \%$ positive, characterizing a good understanding between ontology specialists without knowledge about the domain.

\section{Discussion}

Ontology development is a hard process. The developer needs to create logical links with the content where mind maps contribute to the organization of ideas. The use of methods as 101 is indispensable. Researchers with no experience having guidelines to achieve structured results at their disposal. Once each ontology is unique, a systemic process is key to promising results.

One challenge of dealing with costs is how to estimate real values. The database can be responsible for the cost gap perceived. If the database is constantly updated, the cost estimation can be assertive; with outdated data, it reduces the assertions. As the objective is to support the specialist in the initial stages, it is necessary to avoid misunderstandings.

Another challenge is the understanding of the language OWL and semantic intelligence. Researches working in interdisciplinary projects coming from different 
study area have more challenges. Sharing knowledge with ontology experts was a beneficial decision. Several tips helped to build artifact and learning. Despite [25] describe the method as cumbersome, it is useful to guide the ontology evaluation in the lab, even more with non-experience developers.

\section{Conclusion}

The cost estimation in early phases of NPD is a complex activity which can be supported by intelligent systems. Many variables and uncertainties of the conceptual project make the specialist unsafe to develop an assertive product in a short time. It is presented a case study about an overhead crane for a cost estimate supported by an ontological model in NPD. The artifact was developed using Protégé ontology editor in order to construct and to simulate the model applicability.

The product's final costs being defined at the beginning of the project contribute to decision makings. Components and assemblies had their costs quickly revealed by the artifact with the knowledge reuse. The cost estimation supports the specialist in the begging of the project providing information that appears just at the end of the product development. Assemblies with different component possibilities now instantaneously have a defined cost.

The applicability in an industrial context is possible satisfying the data quantity and abstraction conditions. A complete database increases ontology efficiency since more data is used to estimate the costs of a component or assembly. The ontology developed is the first stage of an ontology applicable in the industry. The structure is the support used to insert more classes, instances, and properties according to the knowledge the user intended.

In this project, the management of the ontology was restricted to the developer, but in the industrial context, it is necessary to analyze who can really work with this tool. To find the answers and generate knowledge, an ontology expert is suitable.

More research could be developed to address the use of machine learning. With each inference, the machine increases the data quantity. With each question, a piece of new information feeds the database in the construction of knowledge. It can be an alternative to avoid repetitive tasks. Another future research is the use of local databases that allow the ontology to access the data and infer remotely. Or, even, the use of Stardog tool [26] instead of the Protégé Sparql plugin. This tool is fed by a virtual database, for example, DBPedia, and makes inferences in the Stardog itself. Approaches that improve humanmachine interaction usually have multiple benefits.

\section{References}

[1] M. Nafisi, M. Wiktorsson and C. Rösiö, Manufacturing Involvement in New Product Development: An Explorative Case Study in Heavy Automotive Component Assembly, Procedia CIRP, Vol. 50, pp. 65-69, 2016.

[2] M. Gopalakrishnan, T. Libby, J. A. Samuels, and D. Swenson, The effect of cost goal specificity and new product development process on cost reduction performance, Accounting, Organ. Soc., vol. 42, pp. 1-11, Apr. 2015.

[3] G. Pahl, W. Beitz, J. Feldhusen, and K.-H. Grote, Engineering Design, 3. Springer London, 2007.

[4] Y. Hooshmand, P. Köhler, and A. Korff-Krumm, Cost Estimation in Engineer-to-Order Manufacturing, Open Eng., vol. 6, no. 1, pp. 22-34, Feb. 2016.

[5] D. Baxter et al., A framework to integrate design knowledge reuse and requirements management in 
engineering design, Robot. Comput. Integr. Manuf., vol. 24, no. 4, pp. 585-593, Aug. 2008.

[6] V. Fortineau, T. Paviot, and S. Lamouri, Improving the interoperability of industrial information systems with description logic-based models-The state of the art, Comput. Ind., vol. 64, no. 4, pp. 363-375, May 2013.

[7] W. J. C. Verhagen, P. Bermell-Garcia, R. E. C. van Dijk, and R. Curran, A critical review of Knowledge-Based Engineering: An identification of research challenges, Adv. Eng. Informatics, Vol. 26, no. 1, pp. 5-15, Jan. 2012.

[8] D. Zhang, D. Hu, Y. Xu, and H. Zhang, A framework for design knowledge management and reuse for Product-Service Systems in construction machinery industry, Comput. Ind., Vol. 63, no. 4, pp. 328-337, May 2012.

[9] X. Zhao, W. J. C. Verhagen, and R. Curran, Estimation of aircraft component production cost using knowledge based engineering techniques, Adv. Eng. Informatics, vol. 29, no. 3, pp. 616-632, Aug. 2015.

[10] M. Mengoni, M. Mandolini, M. Matteucci, and M. Germani, A Scalable 'Design for Costing' Platform: A Practical Case in Ball Valves Industry, Procedia CIRP, vol. 50, pp. 311-317, 2016.

[11] I. O. Sanya and E. M. Shehab, An ontology framework for developing platform-independent knowledge-based engineering systems in the aerospace industry, Int. J. Prod. Res., vol. 52, no. 20, pp. 6192-6215, 2014.

[12] H. Fazlollahtabar and N. Mahdavi-Amiri, Design of a neuro-fuzzy-regression expert system to estimate cost in a flexible jobshop automated manufacturing system, Int. J. Adv. Manuf. Technol., vol. 67, no. 5-8, pp. 1809-1823, Jul. 2013.

[13] M. Relich, Computational Intelligence for Estimating Cost of New Product Development, Found. Manag., vol. 8, no. 1, pp. 21-34, Jan. 2016.

[14] S. Xu, K. Liu, L. C. M. Tang, and W. Li, A framework for integrating syntax, semantics and pragmatics for computer-aided professional practice: With application of costing in construction industry, Comput. Ind., vol. 83, pp. 28-45, Dec. 2016.

[15] N.F. Noy and D. L. McGuinness, Ontology Development 101: A Guide to Creating Your First Ontology, Stanford, 2001.

[16] R. Voltolini, K. Vasconcelos, M. Borsato, and M. Peruzzini, Research and Analysis of Opportunities in Product Development Cost Estimation Through Expert Systems, Advances In Transdisciplinary Engineering, Vol. 7: Transdisciplinary Engineering Methods for Social Innovation of Industry 4.0, 2018, pp. 381-390.

[17] Y. He, Z. Xiang, J. Zheng, Y. Lin, J. A. Overton, and E. Ong, The eXtensible ontology development (XOD) principles and tool implementation to support ontology interoperability, J. Biomed. Semantics, vol. 9, no. 1, pp. 1-10, 2018.

[18] M. Gruninger and M. Fox, Methodology for the Design and Evaluation of Ontologies, Toronto, 1995.

[19] M. Vegetti, L. Roldán, S. Gonnet, H. Leone, and G. Henning, A framework to represent, capture, and trace ontology development processes, Eng. Appl. Artif. Intell., vol. 56, no. July, pp. 230-249, 2016.

[20] M. Fernández, A. Gómez-Pérez, and N. Juristo, Methontology: from ontological art towards ontological engineering, Madrid, 1997.

[21] R. Owen and I. Horváth, Towards Product-Related Knowledge Asset Warehousing in Enterprises, International Symposium on Tools and Methods of Competitive Engineering, 2002, pp. 155-170.

[22] W3C, "Query Forms," 2018. [Online]. Available: https://www.w3.org/TR/rdf-sparqlquery/\#QueryForms. [Accessed: 03-Feb-2018].

[23] S. Lohmann, V. Link, E. Marbach, and S. Negru, WebVOWL: Web-based Visualization of Ontologies, in Knowledge Engineering and Knowledge Management, Springer, Cham, 2015, pp. 154-158.

[24] K. Peffers, T. Tuunanen, M. A. Rothenberger, and S. Chatterjee, A Design Science Research Methodology for Information Systems Research, J. Manag. Inf. Syst., vol. 24, no. 3, pp. 45-77, Dec. 2007.

[25] N. Baliyan and A. Verma, Recent Advances in the Evaluation of Ontology Quality, in: M. D. Lytras, N. Aljohani, E. Damiani, and K. T. Chui, (eds.) Semantic Web Science and Real-World Applications, 1st ed., IGI Global, Hershey, 2019, pp. 326-348.

[26] Stardog: The Enterprise Knowledge Graph Platform. [Online]. Available: https://www.stardog.com/. [Accessed: 10-Nov-2018]. 